\title{
CANONICAL CONFIGURATIONS ASSOCIATED WITH A SURFACE*
}

BY E. P. LANE

1. Introduction. After summarizing briefly for subsequent use some of the theory of the projective differential geometry of a surface referred to its asymptotic net, we next present some very convenient formulas for differentiating the coordinates of a point referred to the local tetrahedron customarily employed when the differential equations of the surface are in Fubini's canonical form. Their use is first illustrated in connection with certain configurations generated by different parts of the local tetrahedron at a variable point on the surface, and then they are applied in a brief study of the first and second canonical surfaces, the former being enveloped by the canonical plane and the latter being generated by the canonical point. Finally, the general theory of a pencil of conjugate nets on a surface is applied to a new covariant pencil of conjugate nets, and a sequence of such pencils is constructed.

2. Analytic Foundations. In ordinary projective space the four homogeneous coordinates $x^{(1)}, \cdots, x^{(4)}$ of a point $P$ on an analytic non-ruled surface $S$ are functions of two independent variables $u, v$. If the asymptotic net is parametric and if the proportionality factor of the coordinates is suitably chosen, the functions $x$ satisfy two partial differential equations in Fubini's canonical form

$$
\left\{\begin{array}{l}
x_{u u}=p x+\theta_{u} x_{u}+\beta x_{v}, \\
x_{v v}=q x+\gamma x_{u}+\theta_{v} x_{v},
\end{array} \quad[\theta=\log (\beta \gamma)]\right.
$$

\footnotetext{
* Presented to the Society, September 6, 1928.
} 
The most general transformation of proportionality factor and parameters preserving this canonical form is

$$
x=\text { const. } \bar{x}, \quad \bar{u}=U(u), \quad \bar{v}=V(v) .
$$

A point $y$ given by an expression of the form

$$
y=x_{1} x+x_{2} x_{u}+x_{3} x_{v}+x_{4} x_{u v}
$$

is said to have local coordinates $x_{1}, \cdots, x_{4}$ referred to the local tetrahedron $x, x_{u}, x_{v}, x_{u v}$ with suitably chosen unit point. The vertices of this tetrahedron are covariant points; in fact the line $x x_{u v}$ is the projective normal and the line $x_{u} x_{v}$ is the reciprocal polar of this line with respect to the quadric of Lie; the vertex $x_{u v}$ is the point where the tangent of the curve $u=$ const. on the surface generated by the point $x_{u}$ meets the projective normal. The effect of (2) on the local coordinates is the change of unit point given by

$$
\bar{x}_{1}=x_{1}, \quad \bar{x}_{2}=U^{\prime} x_{2}, \quad \bar{x}_{3}=V^{\prime} x_{3}, \quad \bar{x}_{4}=U^{\prime} V^{\prime} x_{4},
$$

accents indicating differentiation with respect to the var. iables of the functions concerned.

The equation of the canonical plane of $S$ at $P$ is

$$
\phi x_{2}-\psi x_{3}=0,
$$

where

$$
\phi=\frac{\partial}{\partial u} \log \beta \gamma^{2}, \quad \psi=\frac{\partial}{\partial v} \log \beta^{2} \gamma .
$$

And the coordinates of the canonical point are $(0, \psi,-\phi, 0)$.

3. The Derivatives of the Local Coordinates. We shall first give some formulas for the local coordinates of the two firstorder derivative points of the point y given by (3). By easy calculation we find

$$
\left\{\begin{array}{l}
y_{u}=y_{1} x+y_{2} x_{u}+y_{3} x_{v}+y_{4} x_{u v} \\
y_{v}=z_{1} x+z_{2} x_{u}+z_{3} x_{v}+z_{4} x_{u v}
\end{array}\right.
$$


where

$$
\left\{\begin{array}{l}
y_{1}=x_{1 u}+p x_{2}+\left(p_{v}+\beta q\right) x_{4}, \\
y_{2}=x_{2 u}+x_{1}+\theta_{u} x_{2}+\left(\beta \gamma+\theta_{u v}\right) x_{4}, \\
y_{3}=x_{3 u}+\beta x_{2}+\pi x_{4}, \\
y_{4}=x_{4 u}+x_{3}+\theta_{u} x_{4}, \\
z_{1}=x_{1 v}+q x_{3}+\left(q_{u}+\gamma p\right) x_{4}, \\
z_{2}=x_{2 v}+\gamma x_{3}+\chi x_{4}, \\
z_{3}=x_{3 v}+x_{1}+\theta_{3} x_{v}+\left(\beta \gamma+\theta_{u v}\right) x_{4}, \\
z_{4}=x_{4 v}+x_{2}+\theta_{v} x_{4},
\end{array}\right.
$$

the functions $\pi, \chi$ being defined by

$$
\pi=p+\beta \psi, \quad \chi=q+\gamma \phi .
$$

It occasionally happens that we have the equation in local coordinates of a surface covariantly connected with a point $P$ of $S$ and wish to find the envelope of this surface as $P$ varies on $S$. The usual method of procedure is to differentiate partially with respect to $u$ and with respect to $v$ in the equation and then to solve the two resulting equations with the original to obtain the points of contact of the surface with its envelope. We shall now obtain the differentiation formulas to be used in such a situation.

The coordinates of a point $\bar{x}$ on $S$ near $P_{x}$ are given* by the expansions

$$
\begin{cases}x_{1}=1+\cdots, & x_{2}=\Delta u+\cdots, \\ x_{3}=\Delta v+\cdots, & x_{4}=0+\cdots .\end{cases}
$$

And the coordinates $y, z, w$ of the corresponding points $\bar{x}_{u}, \bar{x}_{v}, \bar{x}_{u v}$ near $x_{u}, x_{v}, x_{u v}$ respectively are given in order by the power series

* Stouffer and Lane, Recent developments in projective differential geometry, this Bulletin, vol. 34 (1928), pp. 453-472. See part B, equation 2. 


$$
\begin{gathered}
\left\{\begin{array}{l}
y_{1}=p \Delta u+\cdots, \quad y_{2}=1+\theta_{u} \Delta u+\cdots, \\
y_{3}=\beta \Delta u+\cdots, \quad y_{4}=\Delta v+\cdots,
\end{array}\right. \\
\left\{\begin{array}{l}
z_{1}=q \Delta v+\cdots, z_{2}=\gamma \Delta v+\cdots, \\
z_{3}=1+\theta_{v} \Delta v+\cdots, z_{4}=\Delta u+\cdots ;
\end{array}\right. \\
\left\{\begin{array}{l}
w_{1}=(p v+\beta q) \Delta u+\left(q_{u}+\gamma p\right) \Delta v+\cdots, \\
w_{2}=\left(\beta \gamma+\theta_{u v}\right) \Delta u+\chi \Delta v+\cdots, \\
w_{3}=\pi \Delta u+\left(\beta \gamma+\theta_{u v}\right) \Delta v+\cdots, \\
w_{4}=1+\theta_{u} \Delta u+\theta_{v} \Delta v+\cdots
\end{array}\right.
\end{gathered}
$$

If a point has coordinates $x_{1}, \cdots, x_{4}$ referred to the original tetrahedron $x, x_{u}, x_{v}, x_{u v}$ and coordinates $\bar{x}_{1}, \cdots, \bar{x}_{4}$ referred to the neighboring tetrahedron $\bar{x}, \bar{x}_{u}, \bar{x}_{v}, \bar{x}_{u v}$, the transformation between the tetrahedrons is found to be given by

$$
\left\{\begin{aligned}
\bar{x}_{1}= & x_{1}-x_{2} p \Delta u-x_{3} q \Delta v-x_{4}\left[\left(p_{v}+\beta q\right) \Delta u\right. \\
& \left.+\left(q_{u}+\gamma p\right) \Delta v\right]+\cdots \\
\bar{x}_{2}= & -x_{1} \Delta u+x_{2}\left(1-\theta_{u} \Delta u\right)-x_{3} \gamma \Delta v \\
& -x_{4}\left[\left(\beta \gamma+\theta_{u v}\right) \Delta u-\chi \Delta v\right]+\cdots \\
\bar{x}_{3}= & -x_{1} \Delta v-x_{2} \beta \Delta u+x_{3}\left(1-\theta_{v} \Delta v\right) \\
& -x_{4}\left[\pi \Delta u+\left(\beta \gamma+\theta_{u v}\right) \Delta v\right]+\cdots \\
\bar{x}_{4}= & -x_{2} \Delta v-x_{3} \Delta u+x_{4}\left(1-\theta_{u} \Delta u-\theta_{v} \Delta v\right)+\cdots .
\end{aligned}\right.
$$

Holding $v=$ const. and taking the limit in these formulas as $\Delta u$ approaches zero (having first transposed certain terms and then divided by $\Delta u$ ), and then interchanging the roles of $u$ and $v$ and repeating the process, we obtain the desired formulas for the differentiation of the local coordinates:

$$
\left\{\begin{array}{l}
x_{1 u}=-p x_{2}-\left(p_{v}+\beta q\right) x_{4}, \\
x_{2 u}=-x_{1}-\theta_{u} x_{2}-\left(\beta \gamma+\theta_{u v}\right) x_{4} \\
x_{3 u}=-\beta x_{2}-\pi x_{4} \\
x_{4 u}=-x_{3}-\theta_{u} x_{4} \\
x_{1 v}=-q x_{3}-\left(q_{u}+\gamma p\right) x_{4} \\
x_{2 v}=-\gamma x_{3}-\chi x_{4} \\
x_{3 v}=-x_{1}-\theta_{v} x_{3}-\left(\beta \gamma+\theta_{u v}\right) x_{4} \\
x_{4 v}=-x_{2}-\theta_{v} x_{4} .
\end{array}\right.
$$


4. Applications to the Local Tetrahedron. It follows from statements in $\$ 2$ that the loci of the points $x_{u}, x_{v}, x_{u v}$ are three surfaces covariant to $S$. Moreover these surfaces and $S$ have their points in correspondence, corresponding points being those that have the same curvilinear coordinates. Without giving details of the calculations we shall simply remark that if we place $x_{1}=x, x_{2}=x_{u}, x_{3}=x_{v}, x_{4}=x_{u v}, u^{1}=u$, $u^{2}=v$, it is easy to determine a system of linear differential equations* of the form

$$
\frac{\partial x_{i}}{\partial u^{p}}=\sum_{j=1}^{4} a_{i j p} x_{j}, \quad(i=1, \cdots, 4 ; p=1,2),
$$

satisfied by the coordinates of these four points. Then the results of D. Sun on quadruples of surfaces with points in correspondence can be applied to these four surfaces. Morecver, each of the three pairs of opposite edges of the local tetrahedron generates two congruences with generators in correspondence, to which the results of $\mathrm{A}$. Cook on pairs of congruences with generators in correspondence can be applied. $\dagger$

The envelopes of the faces of the local tetrahedron are again a quadruple of surfaces with points in correspondence. It is geometrically obvious, and easily demonstrated by (15), that the face $x_{4}=0$ envelops $S$ itself. The points of contact of the faces $x_{1}=0, x_{2}=0, x_{3}=0$ with their envelopes are found by (15) to have respectively the coordinates

$$
\left\{\begin{array}{l}
{\left[0,-q\left(p_{v}+\beta q\right),-p\left(q_{u}+\gamma p\right), p q\right]} \\
{\left[-\gamma\left(\beta \gamma+\theta_{u v}\right), 0,-\chi, \gamma\right]} \\
{\left[-\beta\left(\beta \gamma+\theta_{u v}\right),-\pi, 0, \beta\right] .}
\end{array}\right.
$$

If, for instance, the last two of these points are joined we obtain a generator of a congruence covariant to $S$, and so a

* Lane, The projective differential geometry of systems of linear homogeneous differential equations of the first order, Transactions of this Society, vol. 30 (1928), pp. 785-796. See $\$ 5$.

† Sun, Cook; Chicago Doctoral Dissertations (1928-29). 
wide variety of possibilities for the construction of covariant configurations is suggested.

5. Applications to the Canonical Surfaces. If $\psi=0$, the canonical point coincides with the point $x_{v}$. Let us suppose $\psi \neq 0$, and place

$$
\lambda=\phi / \psi .
$$

Then the local coordinates of the canonical point are $(0,1$, $-\lambda, 0)$ and the equation of the canonical plane is

$$
\lambda x_{2}-x_{3}=0 .
$$

Applying (15) to (19) we find that the envelope of the canonical plane, as $u, v$ vary, is a surface, which we shall call the first canonical surface $S_{1}$, and whose point of contact with the plane is given by the formulas

$$
\left\{\begin{aligned}
& x_{1}=-\left(\beta \gamma+\theta_{u v}\right) x_{4}+\pi\left(\lambda_{v}+\theta_{v} \lambda-\gamma \lambda^{2}\right) \\
& \quad+\chi \lambda\left(\lambda_{u}+\beta-\theta_{u} \lambda\right) \\
& x_{2}=-\pi+\chi \lambda^{2}, \quad x_{3}=\lambda x_{2} \\
& x_{4}=\lambda_{u}+\lambda \lambda_{v}+\beta-\theta_{u} \lambda+\theta_{v} \lambda^{2}-\gamma \lambda^{3}
\end{aligned}\right.
$$

The locus of the canonical point, as $u, v$ vary, is the second canonical surface $S_{2}$, whose tangent plane can be shown by means of (8) to have coordinates given by

(21) $\left\{\begin{array}{l}u_{1}=\lambda_{u}+\lambda \lambda_{v}-\beta-\theta_{u} \lambda+\theta_{v} \lambda^{2}+\gamma \lambda^{3}, \\ u_{2}=\lambda u_{3}, \quad u_{3}=p-q \lambda^{2}, \\ u_{4}=p\left(\lambda_{v}+\theta_{v} \lambda+\gamma \lambda^{2}\right)+q \lambda\left(\lambda_{u}-\beta-\theta_{u} \lambda\right) .\end{array}\right.$

It will be observed that the two surfaces $S_{1}, S_{2}$ correspond in the dualistic transformation that converts each point $P$ of $S$ into the tangent plane of $S$ at $P$. Analytically this kind of duality* is represented in the present notation by the transformation

* Lane, The correspondence between the tangent plane of a surface and its point of contact, American Journal of Mathematics, vol. 48 (1926), p. 204. 
(22) $x_{1}=-\left(\beta \gamma+\theta_{u v}\right) u_{1}+u_{4}, x_{2}=-u_{3}, x_{3}=-u_{2}, x_{4}=u_{1}$ accompanied by the substitution

$$
\left(\begin{array}{rrrrr}
\beta & \gamma & p & q & d v / d u \\
-\beta & -\gamma & \pi & \chi & -d v / d u
\end{array}\right)
$$

We shall make a few remarks about $S_{2}$, understanding that similar remarks, mutatis mutandis, can be made about $S_{1}$.

The tangent plane of $S_{2}$ passes through $P_{x}$ in case

$$
\lambda_{u}+\lambda \lambda_{v}-\beta-\theta_{u} \lambda+\theta_{v} \lambda^{2}+\gamma \lambda^{3}=0,
$$

and then its line of intersection with the tangent plane of $S$ is the second canonical tangent joining $P_{x}$ to the canonical point. In this case the canonical curves of the first kind $d v-\lambda d u=0$ are dual union curves of the reciprocal of the projective normal congruence, so that the ray-point of the first canonical curve through $P_{x}$ lies on the reciprocal $x_{4}=x_{1}=0$ of the projective normal, being in fact the canonical point itself.

The tangent plane of $S_{2}$ meets the tangent plane of $S$ in the reciprocal of the projective normal in case

$$
p-q \lambda^{2}=0
$$

being in this case the plane $x_{1}=0$. Then the canonical curves

$$
d v^{2}-\lambda^{2} d u^{2}
$$

correspond to the developables of the reciprocal of the projective normal congruence, which is therefore harmonic to the conjugate net of canonical curves.

The locus of the canonical point is a curve in case (24) and (25) are satisfied simultaneously.

6. The Canonical Pencil of Conjugate Nets. A pencil of conjugate nets on a surface is the class of $\infty^{1}$ conjugate nets every one of which has the property that at every point of the surface its two tangents form with the tangents of a fundamental conjugate net the same cross ratio. The covariant pencil of conjugate nets determined by the canonical 
conjugate net (26) will be called the canonical pencil. The differential equation of a general net of this pencil is

$$
d v^{2}-\lambda^{2} h^{2} d u^{2}=0 \quad(h=\text { const. }) .
$$

The envelope of the osculating planes at $P$ of all curves of the canonical pencil that pass through $P$ is the axis-plane* cone

$$
u_{1}=u_{2} u_{3} m-\beta u_{3}^{8}-\gamma u_{2}^{3}=0
$$

where

$$
m=2 u_{4}-\left(\theta_{u}-\lambda_{u} / \lambda\right) u_{3}-\left(\theta_{v}+\lambda_{v} / \lambda\right) u_{2} .
$$

The cusp-axis of this cone joins $P_{x}$ to the point

$$
\left(0,-\theta_{v}-\lambda_{v} / \lambda,-\theta_{u}+\lambda_{u} / \lambda, 2\right),
$$

which lies in the canonical plane in case

$$
\lambda\left(\theta_{v}+\lambda_{v} / \lambda\right)=\theta_{u}-\lambda_{u} / \lambda .
$$

Let us suppose that (30) is not satisfied. Then the plane $\lambda_{1} x_{2}-x_{3}=0$, where

$$
\lambda_{1}=\frac{\theta_{u}-\lambda_{u} / \lambda}{\theta_{v}+\lambda_{v} / \lambda}
$$

contains the cusp-axis and the projective normal. The curves enveloped by the line of intersection of this plane with the tangent plane of $S$, together with their conjugates, form a new covariant conjugate net

$$
d v^{2}-\lambda_{1}^{2} d u^{2}=0
$$

and the process can be repeated indefinitely. In this way we obtain a sequence of covariant conjugate nets.

The University of Chicago

* Lane, $A$ general theory of conjugate nets, Transactions of this Society, vol. 23 (1922), p. 283. 